\title{
Susceptibilidad antibiótica de Helicobacter pylori: un estudio de prevalencia en pacientes con dispepsia en Quito, Ecuador
}

\section{Susceptibility of Helicobacter Pylori to Antibiotics: a Study of Prevalence in Patients with Dyspepsia in Quito, Ecuador}

Jorge Reyes Ch., ${ }^{1}$ Katherine Guzmán, ${ }^{2}$ Eduardo Morales, ${ }^{2}$ José Villacís, ${ }^{3}$ Galo Fernando Pazmiño Quirós, ${ }^{4}$ Rosa Eugenia Pacheco Tigselema, ${ }^{5}$ Luis Santiago Escalante. ${ }^{2}$

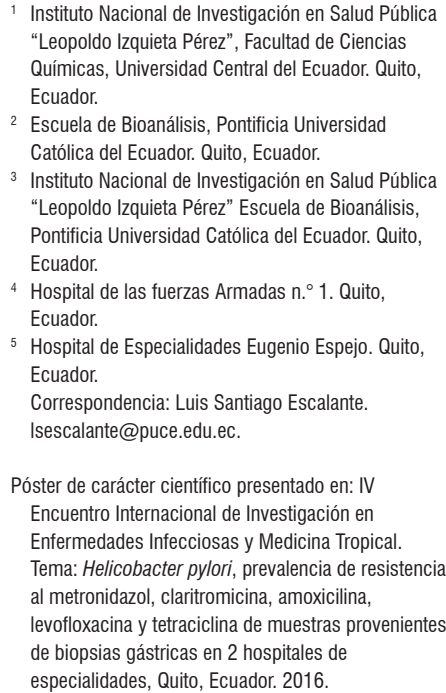

2 Escuela de Bioanálisis, Pontificia Universidad Católica del Ecuador. Quito, Ecuador.

3 Instituto Nacional de Investigación en Salud Pública "Leopoldo Izquieta Pérez" Escuela de Bioanálisis, Pontificia Universidad Católica del Ecuador. Quito, Ecuador.

${ }^{4}$ Hospital de las fuerzas Armadas n. ${ }^{\circ}$ 1. Quito, Ecuador.

5 Hospital de Especialidades Eugenio Espejo. Quito, Ecuador.

Correspondencia: Luis Santiago Escalante. Isescalante@puce.edu.ec.

Póster de carácter científico presentado en: IV Encuentro Internacional de Investigación en Enfermedades Infecciosas y Medicina Tropical. Tema: Helicobacter pylori, prevalencia de resistencia al metronidazol, claritromicina, amoxicilina, levofloxacina y tetraciclina de muestras provenientes de biopsias gástricas en 2 hospitales de especialidades, Quito, Ecuador. 2016.

\begin{abstract}
Resumen
Introducción: el Helicobacter pylori se asocia con patologías gastrointestinales, el incremento en la resistencia a los antibióticos utilizados para su erradicación es alarmante a nivel mundial. En este estudio se determinó la susceptibilidad a 5 antibióticos utilizados en la terapia de erradicación de $H$. pylori aislado de una población adulta con dispepsia recurrente en Quito, Ecuador. Materiales y métodos: previa aceptación del consentimiento informado, se tomaron biopsias de cuerpo y fondo gástrico de 210 pacientes con dispepsia y mediante cultivo se recuperaron los aislados de $\mathrm{H}$. pylori identificado mediante pruebas bioquímicas. La susceptibilidad al metronidazol, claritromicina, amoxicilina, tetraciclina y levofloxacina se realizó por concentración mínima inhibitoria (CMI). Todos los cultivos se correlacionaron con el estudio histopatológico. Resultados: se recuperaron 89 aislados de $H$. pylori. Se obtuvo un kappa de 0,9 entre el cultivo y el estudio histopatológico. El porcentaje de cepas con resistencia antibiótica fue: metronidazol (63\%), claritromicina $(66 \%)$, amoxicilina (43\%), tetraciclina (36\%) y levofloxacina (54\%). Conclusión: estos hallazgos demuestran la alta resistencia a los antibióticos usados para la erradicación de H. pylori, varios factores como el consumo indiscriminado de antibióticos, terapia previa, entre otros podrían estar involucrados.
\end{abstract}

\section{Palabras clave}

Helicobacter pylori; resistencia antibiótica; concentración mínima inhibitoria.

\begin{abstract}
Introduction: Worldwide, helicobacter pylori is associated with gastrointestinal pathologies, but increasing resistance to antibiotics used for its eradication is causing alarm. This study determined susceptibility of $\mathrm{H}$. pylori to five antibiotics used in eradication therapy in an adult population with recurrent dyspepsia in Quito, Ecuador. Materials and methods: After patients provided informed consent, biopsies were taken from the gastric corpus and fundus of 210 patients with dyspepsia. H. pylori isolates identified by biochemical tests were recovered from cultures of biopsy samples. Minimal inhibitory concentrations (MIC) of metronidazole, clarithromycin, amoxicillin, tetracycline and levofloxacin were tested to indicate susceptibility. All cultures were correlated with the histopathological study. Results: H. pylori isolates were recovered from 89 cultures. A kappa of 0.9 was obtained between the culture and the histopathological study. The percentage of strains with antibiotic resistance were $63 \%$ for metronidazole, $66 \%$ for clarithromycin, $43 \%$ for amoxicillin, $36 \%$ for tetracycline and $54 \%$ for levofloxacin. Conclusion: These findings demonstrate high levels of resistance to the antibiotics used for eradication of $\mathrm{H}$. pylori. Several factors including indiscriminate consumption of antibiotics and previous therapy may be involved.
\end{abstract}

Keywords

Helicobacter pylori; antibiotic resistance; minimal inhibitory concentration. 


\section{INTRODUCCIÓN}

El Helicobacter pylori es una bacteria bacilar gramnegativa que habita en la mucosa gástrica, se asocia con enfermedades gastrointestinales inflamatorias como úlcera péptica, gastritis crónica y neoplásicas como los linfomas tipo MALT (tejido linfoide asociado a mucosas) donde la producción de citotoxinas como la asociada al gen $\mathrm{A}$ (cagA) y la vacuolizante $(\operatorname{vacA})$ han sido asociados $(1,2) . H$. pylori se adquiere durante la niñez, permanece de forma asintomática durante muchos años y el contacto cercano es un factor de riesgo $(3,4)$. Estudios de prevalencia han demostrado que la presencia de $H$. pylori en personas adultas en países en vías de desarrollo supera el $70 \%$ (5), mientras que en países desarrollados oscila entre el $24 \%$ y el $42 \%$ (6).

La terapia de erradicación de $H$. pylori basada en antibióticos asociados con un inhibidor de bomba de protones alcanza hasta un $80 \%$ de efectividad; sin embargo, existe un porcentaje de fallo terapéutico debido principalmente por resistencia a los antibióticos (7), la utilización del cultivo y el antibiograma para el desarrollo de guías terapéuticas con base en datos de resistencia bacteriana locales son una estrategia adecuada $(7,8)$.

El estudio pretende determinar la prevalencia de la resistencia antibiótica en aislados clínicos de $H$. pylori de pacientes mayores de 18 años con dispepsia recurrente.

\section{MATERIALES Y MÉTODOS}

\section{Pacientes}

El estudio se llevó acabo en 2 hospitales públicos de especialidades (aproximadamente 400 camas cada uno) de la ciudad de Quito, Ecuador, en un período de 6 meses, se seleccionaron a los pacientes mayores de 18 años con síntomas de dispepsia recurrente, que han aceptado ingresar al estudio mediante la firma del consentimiento informado y que no hayan tomado tratamiento antibiótico por lo menos 5 días antes de la endoscopía digestiva alta (EDA).

\section{Aislamiento e identificación de $\boldsymbol{H}$. pylori}

Mediante EDA se tomaron 2 biopsias gástricas, una de fondo y otra de antro, colocadas en un frasco de tapa rosca con $1 \mathrm{~mL}$ de solución salina $0,9 \%$ estéril y transportadas durante las 2 primeras horas (9) al laboratorio de la Escuela de Bioanálisis de la Pontificia Universidad Católica del Ecuador (PUCE) y al laboratorio de referencia nacional de resistencia a los antimicrobianos del Instituto Nacional de Investigación en Salud Pública Leopoldo Izquieta Pérez (INSPI "LIP") para su control y supervisión. Luego de maceradas, se estriaron en agar base columbia (Oxoid UK) suplementado con sangre de cordero al $7 \%$, vancomicina 10 $\mathrm{mg} / \mathrm{L}$, trimetoprima $5 \mathrm{mg} / \mathrm{L}$, cefsulodina $5 \mathrm{mg} / \mathrm{L}$ y anfotericina $\mathrm{B} 5 \mathrm{mg} / \mathrm{L}$ (DENT', Oxoid UK) siguiendo las instrucciones del fabricante e incubados en atmósfera microaerófila alcanzando $5 \%$ de oxígeno $\left(\mathrm{O}_{2}\right), 10 \%$ de dióxido de carbono $\left(\mathrm{CO}_{2}\right)$ y $85 \%$ de nitrógeno $\left(\mathrm{N}_{2}\right)$ (CampyGen, Oxoid UK) a $37^{\circ} \mathrm{C}$ por 7 días. La identificación de H. pylori se realizó mediante la observación morfológica de la colonia, la coloración de Gram (bacilos gramnegativos), reacción positiva para las pruebas de oxidasa, catalasa y ureasa. Los aislados se almacenaron en infusión cerebro corazón (BHI) (Oxoid UK) con glicerol al 20\% a $-70{ }^{\circ} \mathrm{C}$.

\section{Pruebas de susceptibilidad}

Para alcanzar una turbidez n. ${ }^{\circ} 3$ de la escala de Mc Farland, a partir de cultivo puro se preparó una suspensión bacteriana en caldo BHI (Oxoid UK) incubando durante 3 días en atmósfera microaerófila (CampyGen, Oxoid UK) a 37 ${ }^{\circ} \mathrm{C}(10)$, posteriormente se inoculó en agar Mueller Hinton (Oxoid UK) suplementado con $7 \%$ de sangre de cordero. Se utilizaron tirillas en gradiente de concentración mínima inhibitoria (CMI) para: amoxicilina, metronidazol, tetraciclina, levofloxacina y claritromicina (Liofilchem, Italia), incubando durante 5 días en ambiente microaerofílico (CampyGen, Oxoid UK). Los puntos de corte para la claritromicina se tomaron del Clinical and Laboratory Standards Institute (CLSI), mientras que para el resto de antibióticos se tomaron los puntos de corte epidemiológicos (epidemiological cutoff value [ECV]) del European Committee on Antimicrobial Susceptibility Testing (EUCAST) vigentes. Para el control de calidad se utilizó H. pylori ATCC 43504.

\section{Aspectos éticos}

Previo al estudio, todos los pacientes firmaron el consentimiento informado, indicando los procedimientos que iban a ser realizados con las muestras, los posibles riesgos y beneficios de su participación en la investigación. El trabajo fue aprobado por el Comité de Bioética de cada hospital y de la PUCE.

\section{RESULTADOS}

De las 210 personas involucradas en el estudio, 143 presentaron cierto grado de inflamación gástrica y 2 personas con adenocarcinoma. Se aislaron en total $89(42,4 \%)$ cepas de H. pylori, de las cuales 87 estuvieron asociadas con patología gástrica por histopatología (Tabla 1); 4 cepas no pudieron ser recuperadas para las pruebas de susceptibilidad para: metronidazol, levofloxacina, amoxicilina y tetraciclina, mientras que para la claritromicina se investigaron 78 aislados (Figuras 1 y 2 ). 
Tabla 1. Resultados del estudio citológico de las biopsias gástricas y número de aislados de H. pylori recuperados

\begin{tabular}{lcc}
\hline \multicolumn{1}{c}{ Características histológicas de la mucosa gástrica } & Cantidad de pacientes (\%) & Cantidad de aislados de H. pylori recuperados \\
\hline Gastritis crónica no atrófica & $60(28,6)$ & 15 \\
Gastritis crónica con metaplasia intestinal & $28(12,3)$ & 15 \\
Gastritis crónica folicular & $23(11)$ & 23 \\
Gastritis crónica difusa & $20(9,5)$ & 20 \\
Gastritis crónica erosiva & $8(8,8)$ & 8 \\
Gastritis crónica atrófica & $4(1,9)$ & 4 \\
Adenocarcinoma & $2(1)$ & 2 \\
Negativo & $65(26,9)$ & 2 \\
Total & 210 & 89 \\
\hline
\end{tabular}

El mayor número de cepas que presentaron CMI superiores a los valores de referencia fueron para metronidazol y claritromicina, mientras que el análisis de asociación de antibióticos para los distintos esquemas terapéuticos demostró que el $32 \%$ de los aislados fue resistente a la combinación amoxicilina/claritromicina y un $42 \%$ a la claritromicina/metronidazol (Tabla 2).

\section{DISCUSIÓN}

H. pylori se asocia con procesos inflamatorios en la mucosa gastrointestinal, su prevalencia es alrededor del $70 \%$ al $80 \%$ a nivel mundial, especialmente en países en vías de desarrollo (5). En nuestro estudio se aisló $H$. pylori en 89 pacientes $(42,4 \%)$, solo 2 aislados no se asociaron con inflamación

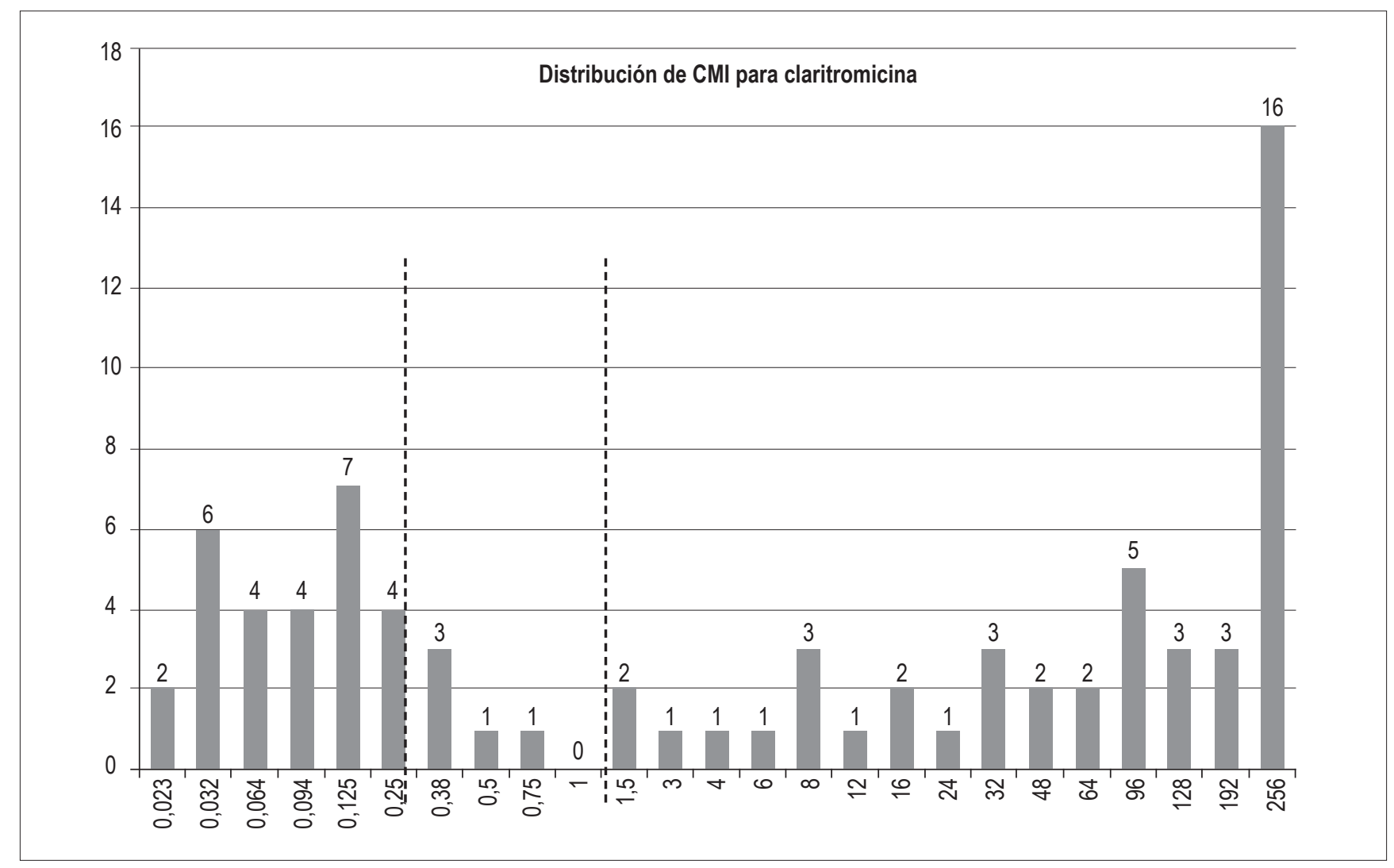

Figura 1. CMI para claritromicina en 78 cepas de H. pylori. Las líneas entrecortadas representan puntos de corte según CLSI 2017. 


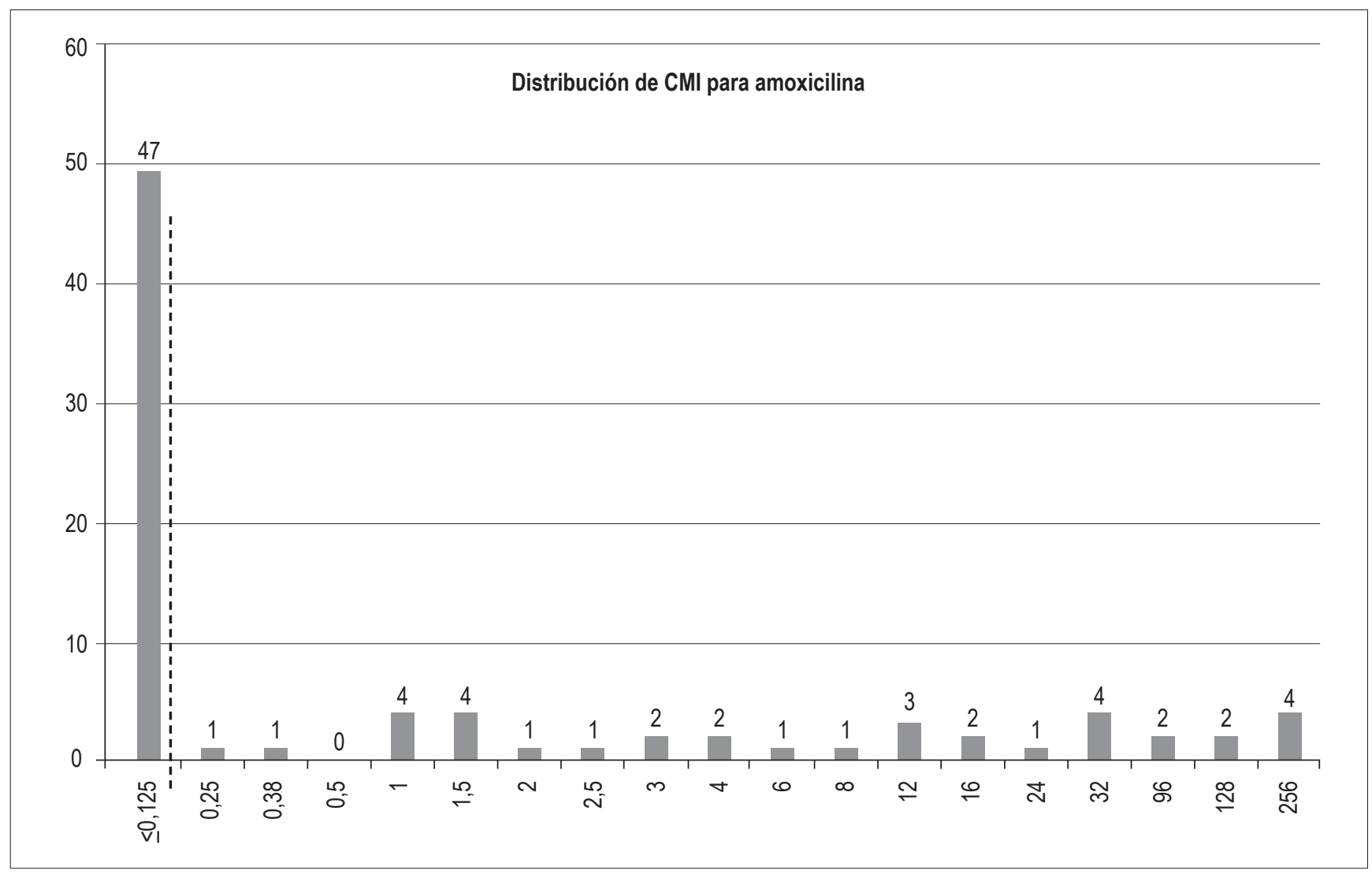

Figura 2. Concentración mínima inhibitoria (CMI) para amoxicilina en 83 cepas de H. pylori. Las líneas entrecortadas representan puntos de corte epidemiológico según EUCAST 2017.

gástrica, y la prevalencia fue más baja que la reportada en otros estudios. Factores como la dificultad de aislar $H$. pylori por medio del cultivo, sitio y calidad de la biopsia gástrica, transporte y condiciones de incubación (11), entre otros pudieron influir en los resultados. En nuestro estudio se seleccionaron las biopsias obtenidas del fondo y antro gástrico como se recomienda (12), mientras que el cultivo se realizó dentro de las 2 horas para evitar la pérdida de la viabilidad de las cepas.

Tanto la resistencia "primaria" asociada con el uso de antibióticos para el tratamiento de infecciones causadas por patógenos distintos a $\mathrm{H}$. pylori, como la resistencia "secundaria" causada por un tratamiento previo con el fin de erradicar H. pylori influyen en su erradicación (13-15). El CLSI establece puntos de corte clínicos para la claritromicina, pero no lo hace para amoxicilina, tetraciclina, metronidazol y levofloxacina. Se ha observado una variación entre estudios epidemiológicos en cuanto a los valores utilizados como puntos de corte, en nuestro estudio se tomaron los puntos de corte epidemiológicos dados por EUCAST y usados en la mayor parte de países de Europa; no obstante, se toma en cuenta que los aislados considerados como resistentes pueden incrementar, así como la falta de eficacia clínica demostrada al utilizarlos (16).

La claritromicina continúa siendo el fármaco recomendado como primera opción para el tratamiento de erradicación (8) de H. pylori. En nuestro estudio se demostró que el $66 \%$ de los aislados presentaba resistencia, valores muy superiores al $12 \%$ reportado para América Latina (17) y al 9,5\% en el Ecuador, reportado en el 2003 (18). No obstante, Raymont y colaboradores demostraron un incremento en la prevalencia de aislados resistentes a la claritromicina llegando al $68 \%$ en pacientes con antecedentes de antibioticoterapia previa (19); un porcentaje similar de resistencia lo demostraron Gao y colaboradores (20).

El consenso de Maastricht IV sobre el uso de macrólidos recomienda que, para el tratamiento empírico de H. pylori, se debe tomar en cuenta la prevalencia local, ya que se debe evitar su uso al observar entre el 15\%-20\% de cepas resistentes y se deben realizar pruebas de susceptibilidad (8); sin embargo, al ser una técnica compleja, pocos laboratorios lo realizan. Entre los mecanismos de resistencia descri- 
Tabla 2. Porcentaje de resistencia a los antibióticos asociados utilizados en el tratamiento de erradicación de H. pylori. Claritromicina: puntos de corte CLSI; para el resto de antibióticos, puntos de corte epidemiológico EUCAST.

\begin{tabular}{lc}
\hline \multicolumn{1}{c}{ Antibióticos investigados } & $\begin{array}{c}\text { \% de aislados de } \text { H. pylori } \\
\text { resistentes según ECV- } \\
\text { EUCAST y CLSI }\end{array}$ \\
\hline Levofloxacina & 54 \\
Tetraciclina & 36 \\
Metronidazol & 63 \\
Claritromicina & 66 \\
Amoxicilina & 43 \\
\hline \multicolumn{1}{c}{ Combinación de antibióticos } & Cantidad de aislados de \\
& H. pylori resistentes (\%) \\
\hline Amoxicilina/claritromicina & $26(32)$ \\
Metronidazol/claritromicina & $32(42)$ \\
Amoxicilina/levofloxacina & $26(32)$ \\
\hline
\end{tabular}

tos a la claritromicina están principalmente las mutaciones en el gen $23 S$ rRNA y presencia de bombas de flujo (21, 22). Una deficiencia de nuestro estudio fue no investigar estos mecanismos.

La amoxicilina es otro antibiótico de primera línea dentro de la terapia triple. En nuestro estudio se encontró que el $43 \%$ de cepas presenta una $\mathrm{CMI} \geq 0,125$, clasificadas como resistentes, un porcentaje elevado si se toma en cuenta que para América Latina se encuentra entre el 0\%-39\%; y aún más para el Ecuador, que en años anteriores no se superaba el $4 \%$ $(17,18)$. No obstante, similar a lo que ocurre con el uso de la claritromicina, Nishizawa y colaboradores demostraron que, luego de varios intentos fallidos de erradicación utilizando amoxicilina dentro del esquema terapéutico, se incrementó el número de aislados resistentes del 49,5\% a 72\% (23). En nuestro estudio no se obtuvieron datos sobre el consumo previo de antibióticos relacionados o no a terapia de erradicación; sin embargo, se seleccionaron pacientes con dispepsia recurrente y que posiblemente recibieron terapéutica antimicrobiana previa. Una resistencia del $63 \%$ al metronidazol se encuentra dentro del rango reportado para América Latina (12,5\%-95\%) y para Ecuador del $80,9 \%(17,18)$.

Más de $30 \%$ de cepas presenta resistencia simultáneamente a 2 antibióticos usados para la terapia de erradicación, sea claritromicina o levofloxacina.

El uso previo de antibióticos es un factor de riesgo para el incremento de cepas de $H$. pylori resistentes y, consecuentemente, para fallo terapéutico, sea por resistencia primaria o secundaria (24). Se demostró una prevalencia elevada de resistencia a los antibióticos utilizados en el tratamiento de erradicación, a diferencia de los resultados obtenidos en
Ecuador en el año 2003; se recomienda el análisis riesgo/ beneficio cuando se utilice la claritromicina o amoxicilina como tratamiento empírico en pacientes con dispepsia recurrentes o que posiblemente ya recibieron tratamiento previo, junto con la realización de pruebas de susceptibilidad.

\section{Agradecimientos}

Al personal del Hospital de Especialidades Eugenio Espejo y Fuerzas Armadas n. ${ }^{\circ} 1$ de la ciudad de Quito, nuestros agradecimientos sinceros.

\section{Conflicto de intereses}

El proyecto Susceptibilidad antibiótica de Helicobacter pylori en Quito, Ecuador: un estudio de prevalencia en pacientes con dispepsia fue producto del Grant otorgado por la Pontificia Universidad Católica del Ecuador, Quito, código: L13295. Sin embargo, estos resultados no fueron influenciados por el ente patrocinador.

\section{REFERENCIAS}

1. Basso D, Zambon CF, Letley DP, Stranges A, et al. Clinical relevance of Helicobacter pylori cagA and vacA gene polymorphisms. Gastroenterology. 2008;135(1):91-9. https:// doi.org/10.1053/j.gastro.2008.03.041

2. Wang F, Meng W, Wang B, et al. Helicobacter pyloriinduced gastric inflammation and gastric cancer. Cancer Lett. 2014;345(2):196-202. https:// doi.org/ 10.1016/ j.canlet.2013.08.016

3. Malaty HM, Kumagai T, Tanaka E, et al. Evidence from a nine-year birth cohort study in Japan of transmission pathways of Helicobacter pylori infection. J Clin Microbiol. 2000;38(5):1971-3.

4. Rocha GA, Rocha AM, Silva LD, et al. Transmission of Helicobacter pylori infection in families of preschool-aged children from Minas Gerais, Brazil. Trop Med Int Health. 2003;8(11):987-91.

5. Porras C,NodoraJ,Sexton R, etal.EpidemiologyofHelicobacter pylori infection in six Latin American countries (SWOG Trial S0701). Cancer Causes Control. 2013;24(2):209-15.

6. Eusebi LH,Zagari RM, BazzoliF.Epidemiology of Helicobacter pylori infection. Helicobacter. 2014;19 Suppl 1:1-5. https:// doi.org/10.1111/hel.12165

7. Graham DY, Fischbach L. Helicobacter pylori treatment in the era of increasing antibiotic resistance. Gut. 2010;59(8):1143-53.

8. Malfertheiner P, Megraud F, O’Morain CA, et al. Management of Helicobacter pylori infection-the Maastricht IV/ Florence Consensus Report. Gut. 2012;61(5):646-64. https://doi.org/10.1136/gutjnl-2012-302084

9. Ndip RN, Malange Takang AE, Ojongokpoko JE, et al. Helicobacter pylori isolates recovered from gastric biopsies 
of patients with gastro-duodenal pathologies in Cameroon: current status of antibiogram. Trop Med Int Health. 2008;13(6):848-54. https:// doi.org/ 10.1111/ j.13653156.2008.02062.x

10. Ogata SK, Gales AC, Kawakami E. Antimicrobial susceptibility testing for Helicobacter pylori isolates from Brazilian children and adolescents: comparing agar dilution, E-test, and disk diffusion. Braz J Microbiol. 2015;45(4):1439-48. https://doi.org/10.1590/S1517-83822014000400039

11. Mégraud F, Lehours P. Helicobacter pylori detection and antimicrobial susceptibility testing. Clin Microbiol Rev. 2007;20(2):280-322. https://doi.org/10.1128/ CMR.00033-06

12. El-Zimaity H, Serra S, Szentgyorgyi E, et al. Gastric biopsies: the gap between evidence-based medicine and daily practice in the management of gastric Helicobacter pylori infection. Can J Gastroenterol [Internet]. 2013;27(10):e2530. https://doi.org/10.1155/2013/897423

13. Poon SK, Chang CS, Su J, et al. Primary resistance to antibiotics and its clinical impact on the efficacy of Helicobacter pylori lansoprazole-based triple therapies. Aliment Pharmacol Ther. 2002;16(2):291-6. https://doi. org/10.1046/j.1365-2036.2002.01184.x

14. Pilotto A, Franceschi M, Rassu M, et al. Incidence of secondary Helicobacter pylori resistance to antibiotics in treatment failures after 1-week proton pump inhibitorbased triple therapies: a prospective study. Dig Liver Dis. 2000;32(8):667-72. https:// doi.org/ 10.1016/ S15908658(00)80327-8

15. Lim SG, Park RW, Shin SJ, et al. The relationship between the failure to eradicate Helicobacter pylori and previous antibiotics use. Dig Liver Dis. 2016;48(4):385-90. https:// doi.org/10.1016/j.dld.2015.12.001

16. Alarcón T, Urruzuno P, Martínez MJ, et al. Antimicrobial susceptibility of 6 antimicrobial agents in Helicobacter pylori clinical isolates by using EUCAST breakpoints compared with previously used breakpoints. Enferm Infecc Microbiol Clin. 2017;35(5):278-282. https://doi.org/10.1016/j. eimc.2016.02.010

17. Camargo MC, García A, Riquelme A, et al. The problem of Helicobacter pylori resistance to antibiotics: a systematic review in Latin America. Am J Gastroenterol. 2014;109(4):485-95. https://doi.org/10.1038/ajg.2014.24

18. Debets-Ossenkopp YJ, Reyes G, Mulder J, et al. Characteristics of clinical Helicobacter pylori strains from Ecuador. J Antimicrob Chemother. 2003;51(1):141-5. https://doi.org/10.1093/jac/dkg023

19. Raymond J, Lamarque D, Kalach N, et al. High level of antimicrobial resistance in French Helicobacter pylori isolates. Helicobacter. 2010;15(1):21-7.

20. Gao W, Cheng H, Hu F, et al. The evolution of Helicobacter pylori antibiotics resistance over 10 years in Beijing, China. Helicobacter. 2010;15(5):460-6.

21. Taylor DE, Ge Z, Purych D, et al. Cloning and sequence analysis of two copies of a 23S rRNA gene from Helicobacter pylori and association of clarithromycin resistance with $23 \mathrm{~S}$ rRNA mutations. Antimicrob Agents Chemother. 1997;41(12):2621-8.

22. Hirata K, Suzuki H, Nishizawa T, et al. Contribution of efflux pumps to clarithromycin resistance in Helicobacter pylori. J Gastroenterol Hepatol. 2010;25 Suppl 1:S75-9. https://doi. org/10.1111/j.1440-1746.2009.06220.x

23. Nishizawa T, Suzuki H, Tsugawa H, et al. Enhancement of amoxicillin resistance after unsuccessful Helicobacter pylori eradication. Antimicrob Agents Chemother. 2011;55(6):30124. https://doi.org/10.1128/AAC.00188-11

24. Megraud F, Coenen S, Versporten A, et al. Helicobacter pylori resistance to antibiotics in Europe and its relationship to antibiotic consumption. Gut. 2013;62(1):34-42. https:// doi.org/10.1136/gutjnl-2012-302254 